\title{
Simultaneous Bilateral Anterior Dislocation of Glenohumeral Joint in \\ Elderly Patients with Seizures: A Case Report
}

\section{Kastanis $\mathbf{G}^{1 *}$, Spyrantis $\mathbf{M}^{1}$, Lazanaki $\mathrm{E}^{2}$, Velivasakis $\mathbf{G}^{\mathbf{1}}$, Christoforidis $\mathrm{C}^{1}$ and Pantouvaki $\mathrm{A}^{3}$}

${ }^{1}$ Department of Orthopaedic, General Hospital of Heraklion, Venizeleio, Crete, Greece

${ }^{2}$ Department of Anesthesiology, General Hospital of Heraklion, Venizeleio, Crete, Greece

${ }^{3}$ Department of Physiotherapy, General Hospital of Heraklion, Venizeleio, Crete, Greece

*Corresponding Author: Kastanis G, Department of Orthopaedic, General Hospital of Heraklion, Venizeleio, Crete, Greece.
Received: January 27, 2020

Published: February 18, 2020

(C) All rights are reserved by Kastanis G., et al.

\begin{abstract}
Anterior glenohumeral dislocation is the most common lesion of dislocations that appears in emergency department. Bilateral anterior shoulder dislocation is a rare injury and in majority of cases originates from trauma. We review two cases (a male, 70 years old and a female, 68 years old) with a simultaneous bilateral anterior dislocation of the shoulder after an epileptic seizure during daily activities. Dislocations were diagnosed after detailed clinical and imaging examination. Under general anesthesia closed reduction with Kocher technique was applied in both patients. A percentage more than $10 \%$ of the bilateral anterior dislocation may missed out in the first diagnosis which leads to serious morbidity and loss of function in patients. The aim of this study is to describe two rare cases with simultaneous bilateral anterior glenohumeral dislocation.
\end{abstract}

Keywords: Shoulder; Dislocation; Bilateral Anterior Dislocation; Epileptic Seizures; Elderly

\section{Introduction}

The glenohumeral articulation is the most usual dislocated joint treated in the emergency department and accounts to $85 \%$ of all joint dislocations in human body. The most common type is the anterior dislocation of the shoulder, which appears at a percentage of $95 \%$ following by posterior, superior, and inferior types with or without fractures [1]. The main causes of bilateral simultaneous shoulder dislocation according to the international literature are falls, electrocution, sport activities, seizures and nocturnal hypoglycemia [18]. During seizures posterior dislocation appears most frequently as a result either directly from trauma during epileptic seizure or through drug- induced bone deminarelization [12]. Si- multaneous anterior dislocation of the shoulder during seizures is a rare manifestation and only a few cases have been reported in the literature.

The mechanism of shoulder dislocation differs between the anterior and posterior dislocations. Posterior glenohumeral dislocation appears from maximal involuntary muscle contraction usually after an electric shock or epileptic seizure while anterior dislocation is a result from trauma [3]. Bilateral anterior shoulder dislocation during seizure is very rarely reported and can be easily missed out if patients are not carefully examined in the emergency. Therefore, emergency physicians must be suspicious of the above co-ex- 
istent injuries. Detailed clinical history is necessary and physical examination should be completed with detailed X-Rays views and clinical neurovascular examination.

The aim of this study is to describe a rare manifestation of two elderly patients with simultaneous bilateral anterior dislocation of the shoulder after an epileptic seizure and to analyze the mechanism of injury and therapy.

\section{Case Report}

A 68 year-old woman presented to the emergency department with right chest and pain in both shoulders, 24 hours after an epileptic seizure during walking in the park. The past clinical history of the patient showed hypertension, diabetes mellitus, and status epilepticus. Blood pressure, pulse, and temperature were normal. Physical examination revealed squaring of both shoulder and restricted total range of motion of affected glenohumeral joints. The neurological and vascular examination of both arms appeared without any deficit. The $\mathrm{x}$-Ray control diagnosed bilateral glenohumeral dislocation with no associated fractures (Figure 1(a) and 1(b)). Under general anaesthesia closed reduc $\neg$ tion was performed using the Kocher's technique in both shoulders. Post reduction $x$-ray examination showed reduction of the glenohumeral joint while the neurovascular examination was intact (Figure 2(a) and 2(b)). Both arms immobilized for ten days in a broad arm sling. The patient initiated a rehabilitation program 3 weeks after immobilization ( 3 times a week), with passive and assistive mobilization of affected joints to increase the range of movement, while an active regime of exercises was started 5 weeks later, to enhance the muscle strength. An individualized home exercise regime was also given.

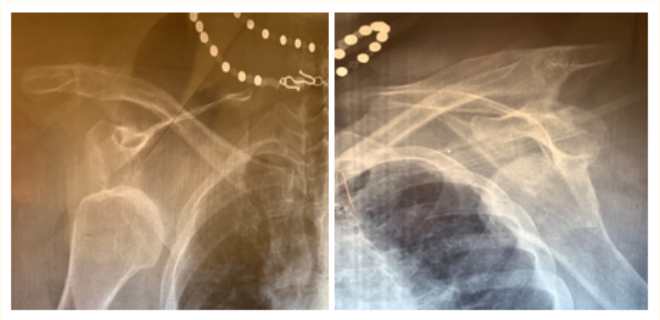

(a)

(b)

Figure 1: X- Rays with bilateral dislocation (a right shoulder, b left shoulder).

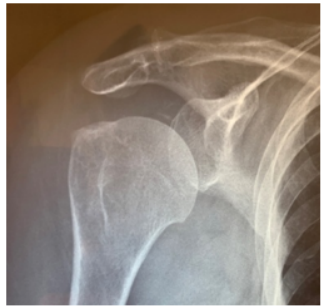

(a)

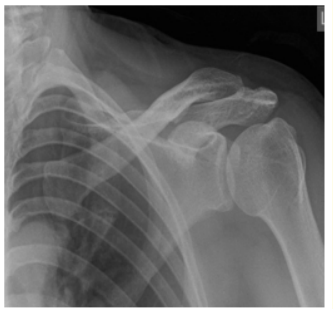

(b)
Figure 2: Post-reduction $\mathrm{x}$-Rays (a right shoulder, b left shoulder).

Follow-up assessment at three and six months showed good functional outcomes in both joints without pain and restricted range of motion of both shoulders. The American Shoulder and Elbow Surgeon Shoulder Score (ASES) was 79,9 at three months and 91,6 at six months post-reduction.

\section{Case Report}

A 70 year old man presented to the emergency department by ambulance after an epileptic seizure with bilateral shoulder pain after a fall from bicycle in countryside. The patient was managed according to ATLS principles, with a GCS of 13 at presentation. The patient's clinical history showed post traumatic seizures. Physical examination showed squaring of both shoulders, disability and restricted total range of motion of affected glenohumeral joints. Blood pressure, pulse, and temperature were normal while neurovascular examination of both upper limbs were intact. Clinical examination was completed with $\mathrm{x}$-Rays of thorax, pelvis and shoulder. These confirmed simultaneous bilateral anterior dislocation of the shoulder without associated fracture (Figure 3). Under general anaesthesia closed reduc $\neg$ tion was performed using the Kocher's technique in both shoulders. Post-reduction x-ray and clinical examination appear successful reduction of the dislocations in both shoulders without neurovascular deficit in both upper arms (Figures 4(a) and 4(b)). Both arms immobilized for ten days in a broad arm sling. The patient initiated a rehabilitation program 3 weeks after immobilization ( 3 times a week), with passive and assistive mobilization of affected joints, while an active regime of exercises was started 5 weeks later, to enhance the muscle strength. An individualized home exercise regime was also given. 


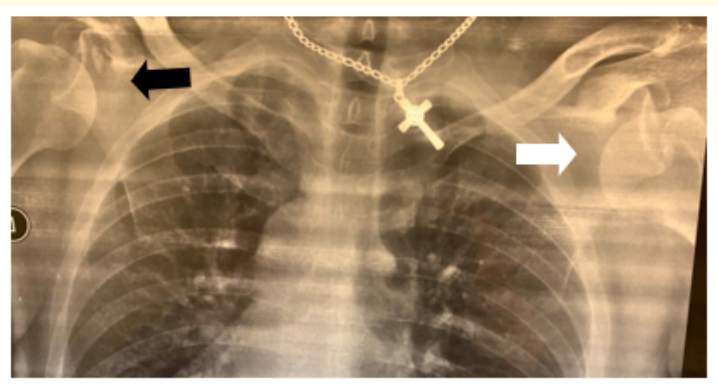

Figure 3: X-rays with bilateral anterior dislocation of glenohumeral joint (black arrow right shoulder and white arrow left shoulder).

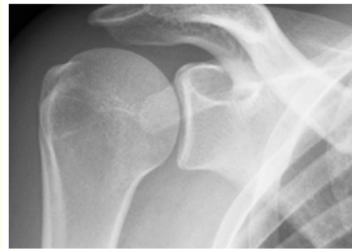

(a)

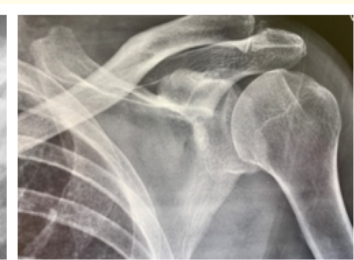

(b)
Figure 4: Post reduction x-rays of Right (a) and Left (b) shoulders.

Finally, follow-up assessment at six months showed good functional outcomes in both joints without pain and restricted range of motion in both shoulders. The American Shoulder and Elbow Surgeon Shoulder Score (ASES) was 74,9 at three months and 93,3 at six months post - reduction.

\section{Discussion}

Simultaneous bilateral glenohumeral dislocations are very rare injuries with the anterior direction appearing rarer than posterior. Mynter H. (1902) first described the bilateral shoulder dislocation in a patient with excessive muscular contraction after camphor overdose [4]. Until today bilateral anterior shoulder dislocations after trauma have been reported as case-reports or small case series in international literature, while only three cases have reported this lesion after a seizure $[2,10,11]$.

Posterior bilateral dislocation is more frequent as a result of electrical shock or seizure leading to strong involuntary muscle contraction, while bilateral anterior shoulder dislocation as a result of trauma [5,6]. Ballesteros., et al. reported that two major causes are responsible for bilateral anterior dislocation of the shoulder: i) trauma that accounts to $50 \%$ and the usual cause is the traction in forward flexion of the shoulder and sport injuries (weight-lifting training, backstroke swimming, chin-up exercise, and horse riding, and ii) muscle contraction (37\%) due to seizures (epileptic, hypoglycemic, toxic or hypoxic) $[7,8]$.

After an epileptic seizure posterior dislocation of the shoulder is the most common lesion because the contraction of weak external rotation and posterior fibers of deltoid muscle predominate by stronger internal rotator, while adduction and internal rotation leads the humeral head to dislocate posteriorly [9]. Bellazzini., et al. described a mechanism of the anterior dislocation during seizure and support that bilateral dislocation may be from trauma of the shoulders striking the floor after a collapse rather than due to the muscle contractions. Loss of consciousness after a seizure will not allow the patient to react and reflexly protect one of his arms by exposing the other [9]. We believe that this mechanism is responsible in both cases we described.

In solitary dislocation the gold standard of therapy is closed reduction during the first six weeks. After this period or in late diagnosed dislocation the open reduction is the treatment of choice with aim to avoid iatrogenic fractures or neurovascular injuries $[8,12]$. In international literature numerous techniques have been described but there is a conflict about which is the more effective one [13]. Techniques of reduction of dislocated shoulder are classified in two categories: leverage (Kocher, Milchs, Cunningham technique) and traction (Hippocrates, Stimson, Eskimo, Spaso, Scapular manipulation technique, Fares method) [14]. All these techniques have advantages and disadvantages. We agree with Dala-Ali that the ideal technique must be simple to perform, effective, requiring minimal force and assistance and being familiar to the physician in the emergency. In our cases we performed Kocher technique and we had immediate successful reduction with no lots of trials and no complications. We believe that the key of reduction is not the predominant technique but the method of sedation or analgesia. General anesthesia offers the advantage of good monitoring control of the patient (history of seizure) with no respiratory or cardiovascular complications. It offers also adequate levels of analgesia and good muscle relaxation, enabling successful realigning of dislocated joint. 
Owens., et al. suggested that an MRI after a first-time dislocation is required to diagnose concomitant injuries (Bankart lesions or rotator cuff tears) [15]. Indications for performing shoulder MRI are young and active patients and first-time dislocation. The aim of the MRI is to decide which surgical method (open or arthroscopic) is necessary to repair the concomitant lesions [16]. When the age of patient is over 40 years old, ultrasound screening has been recommended after first-time dislocation [17]. In our cases patients disagreed to have MRI or Ultrasound screening.

\section{Conclusion}

Simultaneous bilateral anterior dislocation of the shoulder is a rare injury and this rarity must be the suspicion of the physician in the emergency department. Detailed clinical history and physical and imaging examination, complete the diagnosis, notably in elderly patients who have increased risk for late diagnosis. Close reduction with satisfactory anesthesia, in cases with dislocations without fractures, is one way therapy. Additionally, MRI examination should be done to diagnose and repair concomitant injuries, especially in active patients.

\section{Conflict of Interest}

The authors declare that have no conflict of interest.

\section{Bibliography}

1. Devalia KL and Peter VK. "Bilateral anterior shoulder dislocation". Journal of Postgraduate Medicine 51 (2005): 72-73.

2. Tripathy SK., et al. "Simultaneous bilateral anterior shoulder dislocation: Report of two cases and review of the literature". Chinese Journal of Traumatology 14 (2011): 312-315.

3. Nourredine H., et al. "Bilateral anterior shoulder dislocation". BMJ Case Reports (2013). doi: 10.1136/bcr-2012-008308

4. Siu YC and Lui TH. "Bilateral anterior shoulder dislocation". Archives of Trauma Research 3.4 (2014): e18178.

5. Meena S., et al. "Bilateral anterior shoulder dislocation". Journal of Natural Science, Biology and Medicine 4.2 (2013): 499501.

6. Turhan E and Demirel M. "Bilateral anterior glenohumeral disloca $\neg$ tion in a horse rider: a case report and a review of the literature". Archives of Orthopaedic and Trauma Surgery 128.1 (2008): 79-82.
7. Ballesteros R., et al. "Bilateral anterior dislocation of the shoulder: review of seventy cases and proposal of a new etiological mechanical classification". The Journal of Emergency Medicine 44.1 (2013): 269-279.

8. Lasanianos N and Mouzopoulos G. "An undiagnosed bilateral ante $\neg$ rior shoulder dislocation after a seizure: a case report". Cases Journal 1.1 (2008): 342.

9. Bellazzini MA and Deming DA. "Bilateral anterior shoulder dislocartion in a young and healthy man without obvious cause". American Journal of Emergency Medicine 25.6 (2007): 734 .

10. Taneja AK., et al. "Bilateral anterior glenohumeral dislocation and coracoid processes fracture after seizure: acute MRI findings of this rare association". Clinical Imaging 37.6 (2013):1131-1134.

11. Marty B., et al. "Bilateral anterior shoul $\neg$ der dislocation fracture after an epileptic seizure. A case report]". Unfallchirurg 97.7 (1994): 382-384.

12. Ahmad K., et al. "Bilateral spontaneous anterior shoulder dislocation: A missed orthopedic injury mistaken as proximal neuropathy". Chinese Journal of Traumatology 20 (2017): $370 \mathrm{e}-372$.

13. Schneider KN., et al. "Bilateral Anterior Shoulder Dislocation in the Elderly - A Case Report and Review of the Literature". Journal of Orthopaedic Case Report 7.5 (2017): 42-49.

14. Dala-Ali B., et al. "Management of acute anterior shoulder dislocation". British Journal of Sports Medicine 48 (2014): 12091215.

15. Owens BD., et al. "Pathoanatomy of first-time, traumatic, anterior glenohumeral subluxation events". Journal of Bone and Joint Surgery America 92 (2010): 1605.

16. Chuang TY., et al. "Use of preoperative three-dimensional computed tomography to quantify glenoid bone loss in shoulder instability". Arthroscopy 24 (2008): 376-382.

17. Mancini GB., et al. "Anterior shoulder dislocation and injuries of the rotator cuff in patients aged over 40 years. Clinical and sonographic study". Chirurgia Degli Organi di Movimento 86 (2001): 37-44. 
18. Oron A., et al. "Bilateral anterior shoulder dislocation in a paratrooper: A case report". Clinical Researsc in Ortopaedics 1.1 (2018): 1-2.

\section{Assets from publication with us}

- Prompt Acknowledgement after receiving the article

- Thorough Double blinded peer review

- Rapid Publication

- Issue of Publication Certificate

- High visibility of your Published work

Website: https://www.actascientific.com/

Submit Article: https://www.actascientific.com/submission.php

Email us: editor@actascientific.com

Contact us: +919182824667 